\title{
Interaction of a Magnetic Impurity with Strongly Correlated Conduction Electrons
}

\author{
Tom Schork and Peter Fulde \\ Max-Planck-Institut für Physik komplexer Systeme, Bayreuther Str. Hs. 16, D-01187 Dresden, \\ Germany
}

(August 16, 2018)

\begin{abstract}
We consider a magnetic impurity which interacts by hybridization with a system of strongly correlated conduction electrons. The latter are described by a Hubbard Hamiltonian. By means of a canconical transformation the charge degrees of freedom of the magnetic impurity are eliminated. The resulting effective Hamiltonian $H_{\text {eff }}$ is investigated and various limiting cases are considered. If the Hubbard interaction $U$ between the conduction electrons is neglected $H_{\text {eff }}$ reduces to a form obtained by the Schrieffer-Wolff transformation, which is essentially the Kondo Hamiltonian. If $U$ is large and the correlations are strong $H_{\text {eff }}$ is changed. One modification concerns the coefficient of the dominant exchange coupling of the magnetic impurity with the nearest lattice site. When the system is hole doped, there is also an antiferromagnetic coupling to the nearest neighbors of that site involving additionally a hole. Furthermore, it is found that the magnetic impurity attracts a hole. In the case of electron doping, double occupancies are repelled by the impurity. In contrast to the hole-doped case, we find no magnetic coupling which additionally involves a doubly occupied site.
\end{abstract}

71.27.+a, 75.30.Hx, 71.70.Gm

Typeset using REVTEX 


\section{INTRODUCTION}

Recently, heavy-fermion behavior has been observed in the electron-doped cuprate $\mathrm{Nd}_{2-x} \mathrm{Ce}_{x} \mathrm{CuO}_{4}(0.1 \lesssim x \lesssim 0.2)$. 1 Below $0.3 \mathrm{~K}$ a linear specific heat $C_{v}=\gamma T$ is observed with a large Sommerfeld coefficient $\gamma \simeq 4 \mathrm{~J} /\left(\right.$ mole $\left.\mathrm{Nd} \cdot \mathrm{K}^{2}\right)$. In the same temperature regime, the spin susceptibility is found to be independent of the temperature and the SommerfeldWilson ratio is of order unity. These are characteristic features of heavy-fermion excitations.2 However, the characteristic low energy scale of the order of $1 \mathrm{~K}$ which is associated with this behavior is not based on a Kondo lattice effect, as it is the case in other heavy-fermion systems. Rather, it is based on a Zeeman effect, a consequence of the strong electron correlations in the conducting $\mathrm{CuO}_{2}$ planes. charge-transfer insulator instead of a metal, 1 . are therefore coupling to a system of strongly correlated electrons 6 G rather than to weakly or uncorrelated ones.

As a first step towards an understanding of the consequences of the strong correlations, the effect of a single impurity should be investigated. Several authors studied the influence of non-magnetic impurities in systems with strongly correlated electron in order to explain the magnetic properties of the undoped or weakly doped host materials of the high-temperature superconductors (e.g., $\mathrm{La}_{2} \mathrm{CuO}_{4}$ ). The considered impurities stem from substituting $\mathrm{Sr}$ for $\mathrm{La}, \mathrm{B}-10$ or from substitutions in the $\mathrm{CuO}_{2}$ planes, i.e., $\mathrm{Zn}$ for $\mathrm{Cu} 1 \mathrm{1}$ and $\mathrm{S}$ for $\mathrm{O} 14$

Nagaosa et al.15 treated magnetic impurities in an undoped two-dimensional Heisenberg antiferromagnet by adding external spins with a different exchange coupling constant. Nagao et al.16 discussed the influence of both, non-magnetic and magnetic impurity scattering on the spin density wave state and the superconducting state within a Hubbard model for weakly correlated conduction electrons.

In this paper, we study the coupling of a single magnetic impurity to strongly correlated electrons moving on a lattice. The latter are described by a Hubbard Hamiltonian and the total Hamiltonian must, therefore, go beyond that of the single-site Anderson impurity 
model.17 By means of a canonical transformation the charge degrees of freedom of the impurity site are eliminated like by the Schrieffer-Wolff transformation 18 in the case of uncorrelated electrons. Due to the strong correlations of the conduction electrons, new terms appear in the resulting effective Hamiltonian. They are analyzed and interpreted. Special attention is paid to the case of a nearly half-filled conduction band with strong correlations, a situation prevailing, e.g., in $\mathrm{Nd}_{2-x} \mathrm{Ce}_{x} \mathrm{CuO}_{4}$. We believe that the new terms resulting from the correlations of the conduction electrons are of relevance for a number of different systems. If the correlations of the conduction electrons are neglected the effective Hamiltonian reduces again to that of Schrieffer and Wolff.18

In the next section the starting Hamiltonian is defined and the canonical transformation is outlined. The resulting effective Hamiltonian for uncorrelated electrons is discussed in Sec. III. Particularly interesting is the limit of strong correlations. In Sec. IV, we discuss the system at half filling and both, the hole and the electron doped case. Finally, Sec. $\square$ contains a summary of the results and the conclusions.

\section{THE HAMILTONIAN AND ITS TRANSFORMATION}

We describe the strongly correlated conduction electrons in the substrate by a Hubbard Hamiltonian on a hypercubic lattice with unit vectors $x$

$$
H_{c}=-t \sum_{j x, \sigma} c_{j \sigma}^{\dagger} c_{j+x \sigma}+U \sum_{j} n_{j \uparrow} n_{j \downarrow}=H_{t}+H_{U}
$$

The operators $c_{j \sigma}^{\dagger}\left(c_{j \sigma}\right)$ create (destroy) an electron with spin $\sigma$ on site $j$ and $n_{j \sigma}=c_{j \sigma}^{\dagger} c_{j \sigma}$. The magnetic impurity is assumed to contain one orbital (e.g., $4 f$ ), which is either empty or singly occupied. Double occupancies are excluded because of the strong repulsion of electrons in that orbital. The orbital energy is therefore given by

$$
H_{f}=\epsilon_{f} \sum_{\sigma} \hat{f}_{\sigma}^{\dagger} \hat{f}_{\sigma}
$$

with $\hat{f}_{\sigma}^{\dagger}=f_{\sigma}^{\dagger}\left(1-n_{f}\right), n_{f}=\sum_{\sigma} f_{\sigma}^{\dagger} f_{\sigma}$, and $\epsilon_{f}<0$. The operators $f_{\sigma}^{\dagger}$ and $f_{\sigma}$ obey the usual anticommutation relations. For later convenience, we define $r=-\epsilon_{f} / U(>0)$. Finally, we 
assume that the interaction between the $f$ orbital and the conduction electrons is local and described by a hybridization contribution like in the Anderson mode 17

$$
H_{c-f}=V \sum_{\sigma}\left(c_{0 \sigma}^{\dagger} \hat{f}_{\sigma}+\hat{f}_{\sigma}^{\dagger} c_{0 \sigma}\right)
$$

Instead of coupling the $f$ orbital with the site 0 of the Hubbard lattice we could have replaced one site of the lattice by the $f$ orbital. The present choice is motivated by the situation prevailing in $\mathrm{Nd}_{2} \mathrm{CuO}_{4}$.

The total Hamiltonian reads

$$
\begin{aligned}
H= & H_{c}+H_{f}+H_{c-f} \\
= & -t \sum_{j x, \sigma} c_{j \sigma}^{\dagger} c_{j+x \sigma}+U \sum_{j} n_{j \uparrow} n_{j \downarrow}+\epsilon_{f} \sum_{\sigma} \hat{f}_{\sigma}^{\dagger} \hat{f}_{\sigma} \\
& +V \sum_{\sigma}\left(c_{0 \sigma}^{\dagger} \hat{f}_{\sigma}+\hat{f}_{\sigma}^{\dagger} c_{0 \sigma}\right) .
\end{aligned}
$$

From this Hamiltonian we want to derive an effective one, $H_{\text {eff }}$, which acts on the space with a singly occupied $f$ orbital, only. Configurations with an empty $f$ orbital are eliminated by first performing a canonical transformation, thereby eliminating the mixing of configurations with different $f$ orbital occupancies and projecting afterwards onto the space with $n_{f}=1$. The canonical transformation is written as

$$
H_{\text {can }}=e^{S} H e^{-S}
$$

where $S$ is determined by the requirement that $H_{c-f}$ disappears to lowest order in $V$. This leads to

$$
\left[H_{c}+H_{f}, S\right]_{-}=\left(L_{c}+L_{f}\right) S=H_{c-f}
$$

Here we introduced the Liouville operators $L_{c}$ and $L_{f}$ which act on an operator $A$ according to $L_{c} A=\left[H_{c}, A\right]_{-}$and $L_{f} A=\left[H_{f}, A\right]_{-}$, respectively. Equation (2.6) has the formal solution

$$
S=\frac{1}{L_{c}+L_{f}} H_{c-f},
$$

and up to terms of second order in $V$ the Hamiltonian $H_{\text {can }}$ reads 


$$
H_{\mathrm{can}}=H_{c}+H_{f}+\frac{1}{2}\left[S, H_{c-f}\right]_{-} .
$$

Next, we project by means of a projector $P_{f}$ onto the space with $n_{f}=1$. The effective Hamiltonian is then given by

$$
\begin{aligned}
H_{\mathrm{eff}} & =P_{f} H_{\mathrm{can}} P_{f} \\
& =\epsilon_{f}+H_{c}+\frac{V^{2}}{2} \sum_{\sigma, \sigma^{\prime}}\left(\hat{f}_{\sigma^{\prime}}^{\dagger} \hat{f}_{\sigma} c_{0 \sigma^{\prime}} \frac{1}{\epsilon_{f}-L_{c}} c_{0 \sigma}^{\dagger}+\mathrm{hc}\right) .
\end{aligned}
$$

To proceed further we use the expansion

$$
\begin{aligned}
\frac{1}{\epsilon_{f}-L_{c}} c_{0 \sigma}^{\dagger} & =\frac{1}{\epsilon_{f}-L_{U}-L_{t}} c_{0 \sigma}^{\dagger} \\
& =\sum_{\nu=0}^{\infty}\left(\frac{1}{\epsilon_{f}-L_{U}} L_{t}\right)^{\nu} \frac{1}{\epsilon_{f}-L_{U}} c_{0 \sigma}^{\dagger},
\end{aligned}
$$

which is one in powers of $t / \epsilon_{f}$, and terminate it after $\nu=2$. The Liouvilleans $L_{t}$ and $L_{U}$ in Eq. (2.10) are defined by $L_{t} A=\left[H_{t}, A\right]_{-}$and $L_{U} A=\left[H_{U}, A\right]_{-}$, respectively. These terms

are easily evaluated if one expresses $c_{i \sigma}^{\dagger}$ in terms of eigenoperators of $L_{U}$, i.e.,

$$
c_{i \sigma}^{\dagger}=c_{i \sigma}^{\dagger}\left(1-n_{i \bar{\sigma}}\right)+c_{i \sigma}^{\dagger} n_{i \bar{\sigma}}=\hat{c}_{i \sigma}^{\dagger}+\bar{c}_{i \sigma}^{\dagger},
$$

where $\bar{\sigma}=-\sigma$. The last two operators have the eigenvalues 0 and 1 , respectively. The resulting terms of Eq. (2.10) up to second order are listed in Appendix A, where nextnearest neighbor contributions which arise in second order $(\nu=2)$ have been neglected.

Inserting these terms into Eq. (2.9) one obtains $H_{\text {eff }}$ in the form

$$
H_{\mathrm{eff}}=\epsilon_{f}+H_{c}+H^{(0)}+H^{(1)}+H^{(2)} .
$$

The Hamiltonians $H^{(\nu)}$ are of order $t^{\nu}$. The general expressions, which hold for arbitrary values of $U / \epsilon_{f}$, are lengthy and are, therefore, moved to Appendix A. In the following we discuss and list only special limits of them.

\section{LIMIT OF UNCORRELATED CONDUCTION ELECTRONS}

For $U=0(r=\infty)$, we obtain from Eq. (2.12) the result of the conventional SchriefferWolff transformation 18 , i.e., 


$$
\begin{aligned}
H_{\mathrm{eff}}(U=0)= & \epsilon_{f}+\frac{\gamma^{\prime} V^{2}}{2 \epsilon_{f}}\left(2-n_{0}\right) \\
& +\sum_{j x, \sigma} T_{j}^{(0)}\left(c_{j \sigma}^{\dagger} c_{j+x \sigma}+c_{j+x \sigma}^{\dagger} c_{j \sigma}\right) \\
& -\frac{2 \gamma^{\prime} V^{2}}{\epsilon_{f}} \mathbf{S}_{f} \mathbf{s}_{0}+\frac{t V^{2}}{\epsilon_{f}^{2}} \mathbf{S}_{f} \sum_{i(0)}\left(\mathbf{s}_{i 0}+\mathbf{s}_{0 i}\right) .
\end{aligned}
$$

Here, the summation $i(0)$ is over the $Z$ nearest neighbor sites of site $0, \mathbf{S}_{f}$ is the $f$ electron spin, $\mathbf{s}_{0}$ is that of site 0 and

$$
\mathbf{s}_{i 0}=\frac{1}{2} \sum_{\alpha \beta} c_{i \alpha}^{\dagger} \boldsymbol{\sigma}_{\alpha \beta} c_{0 \beta} .
$$

$\boldsymbol{\sigma}$ denote the Pauli matrices.

The last two terms in Eq. (3.1) describe the Kondo coupling between the $f$ spin and

the spin of the conduction electrons 19 Applying $L_{t}$ in Eq. (2.10) results in a delocalization of the interaction to first order in $t$. To second order, we obtain a renormalization factor $\gamma^{\prime}=1+Z t^{2} / \epsilon_{f}^{2}$ for $\mathbf{S}_{f} \mathbf{s}_{0}$. Further delocalization of the interaction is neglected in our approximation, since next-nearest neighbor contributions were not taken into account in the truncated form of Eq. (2.10), see Appendix A. Due to the impurity the electron hopping is reduced between site 0 and its nearest neighbors

$$
T_{j}^{(0)}=-\frac{t}{2}\left(1-\frac{V^{2}}{2 \epsilon_{f}^{2}} \delta_{j 0}\right) .
$$

Finally, there is a one-particle potential, which reduces the probability of having an electron at site 0 , as this would diminish the gain in kinetic energy of the $f$ electron by a virtual hop.

\section{LIMIT OF STRONG CORRELATIONS}

Of particular interest is the limit of strong correlations $(U \gg t)$, which we treat in the following. In contrast to the ordinary Hubbard model (without impurity), the two cases of doping the half-filled system with holes or with electrons are no longer symmetric since we take into account an empty and a singly occupied $f$ orbital only. In the following, we perform a degenerate perturbation theory for both cases and comment on the half-filled case. 


\section{A. Hole doping}

For less than half filling (hole doping), doubly occupied sites are excluded in the limit of strong correlations (large $U$ ). Let $P_{c}$ denote the projector onto the configuration space without doubly occupied sites. Furthermore, set $Q_{c}=1-P_{c}$. The effective Hamiltonian, when reduced to the space of no doubly occupied lattice sites becomes20

$$
\tilde{H}^{(\mathrm{h})}=P_{c} H_{\mathrm{eff}} P_{c}+P_{c} H_{\mathrm{eff}} Q_{c} \frac{1}{E^{(\mathrm{h})}-Q_{c} H_{\mathrm{eff}} Q_{c}} Q_{c} H_{\mathrm{eff}} P_{c}
$$

where $E^{(\mathrm{h})}$ is the ground-state energy. Only those parts of $H_{\text {eff }}$ contribute to the last term to order $U^{-1}$ which generate a single doubly occupied site. Thus,

$$
\tilde{H}^{(\mathrm{h})}=P_{c} H_{\mathrm{eff}} P_{c}-\frac{1}{U} P_{c}\left(H_{t}+H^{(1)}\right) Q_{c}\left(H_{t}+H^{(1)}\right) P_{c}
$$

(see Appendix A). When terms of order $V^{4}$, as well as terms involving three lattice sites are neglected the second term in Eq. (4.2) becomes

$$
\begin{gathered}
-\frac{1}{U} P_{c} H_{\mathrm{eff}} Q_{c} H_{\mathrm{eff}} P_{c}= \\
\frac{2 t^{2}}{U} \sum_{j x}\left(\mathbf{s}_{j} \mathbf{s}_{j+x}-\frac{n_{j} n_{j+x}}{4}\right) \\
+\frac{t^{2} V^{2}\left(1-2 r-2 r^{2}\right)}{\epsilon_{f}^{2} U(1+r)^{2}} \sum_{i(0)}\left(\mathbf{s}_{0} \mathbf{s}_{i}-\frac{n_{0} n_{i}}{4}\right) \\
+\frac{t^{2} V^{2}}{\epsilon_{f}^{2} U(1+r)^{2}} \sum_{i(0)} \mathbf{s}_{f}\left(\mathbf{s}_{0} n_{i}-\mathbf{s}_{i} n_{0}\right),
\end{gathered}
$$

where $\mathbf{s}_{j}$ is the spin at site $j$ and the summation $i(0)$ is restricted over the nearest neighbors of site 0 , only.

One notices that the first term on the right-hand side when added to $P_{c} H_{c} P_{c}$ yields the well-known $t$ - $J$ model, i.e.,

$$
H_{t J}=-t \sum_{j x, \sigma} \hat{c}_{j \sigma}^{\dagger} \hat{c}_{j+x \sigma}+\frac{2 t^{2}}{U} \sum_{j x}\left(\mathbf{s}_{j} \mathbf{s}_{j+x}-\frac{n_{j} n_{j+x}}{4}\right)
$$

with $\hat{c}_{i \sigma}^{\dagger}=c_{i \sigma}^{\dagger}\left(1-n_{i \bar{\sigma}}\right)$. This is expected since the Hubbard Hamiltonian $H_{c}$ reduces to $H_{t J}$ in the strong correlation limit. All terms depending on $V$ describe the interaction of the 
magnetic impurity with the strongly correlated electrons. We regroup the different terms in $\tilde{H}^{(\mathrm{h})}$ and find

$$
\tilde{H}^{(\mathrm{h})}=\Delta E+H_{\text {kin }}+H_{\mathrm{H}}+H_{\mathrm{p}}+H_{\mathrm{K}}+H^{\prime} .
$$

The different contributions are as follows. The term $\Delta E$ describes a constant energy shift:

$$
\Delta E=\epsilon_{f}-\frac{\gamma V^{2}}{2\left(U-\epsilon_{f}\right)},
$$

where $\gamma=1+Z t^{2} /\left(U-\epsilon_{f}\right)^{2}$ is again a renormalization factor. The kinetic energy of the conduction electrons is given by $H_{\text {kin }}$, i.e.,

$$
\begin{aligned}
H_{\mathrm{kin}} & =\sum_{j x, \sigma} T_{j}\left(\hat{c}_{j \sigma}^{\dagger} \hat{c}_{j+x \sigma}+\hat{c}_{j+x \sigma}^{\dagger} \hat{c}_{j \sigma}\right) \\
T_{j} & =-\frac{t}{2}\left(1-\frac{V^{2}\left(2+2 r+r^{2}\right)}{2 \epsilon_{f}^{2}(1+r)^{2}} \delta_{j 0}\right) .
\end{aligned}
$$

One notices that due to the impurity the electron hopping is reduced between site 0 and its nearest neighbors. The interactions between the strongly correlated conduction electrons are described by $H_{\mathrm{H}}$, which is of Heisenberg type

$$
\begin{aligned}
H_{\mathrm{H}} & =\sum_{j x} J_{j}\left(\mathbf{s}_{j} \mathbf{s}_{j+x}-\frac{n_{j} n_{j+x}}{4}\right) \\
J_{j} & =\frac{2 t^{2}}{U}\left(1-\frac{V^{2}}{\left|\epsilon_{f}\right|\left(U-\epsilon_{f}\right)} \delta_{j 0}\right) .
\end{aligned}
$$

Again, the interactions between site 0 and its nearest neighbors are reduced due to the impurity. $\quad H_{\mathrm{p}}$ is a one-particle potential, which describes the attraction of a hole by the impurity as well as its repulsion from a nearest neighbor site

$$
\begin{aligned}
H_{\mathrm{p}}= & -\frac{\eta V^{2}}{2\left|\epsilon_{f}\right|(1+r)}\left(1-n_{0}\right) \\
& +\frac{V^{2} t^{2}}{2 \epsilon_{f}^{2} U(1+r)^{2}} \sum_{i(0)}\left(1-n_{i}\right) \\
\eta= & 2+r+\frac{Z t^{2}}{\epsilon_{f}^{2}(1+r)^{2}}\left(2+7 r+7 r^{2}+r^{3}\right) .
\end{aligned}
$$

The attraction of a hole by the impurity is intuitively clear. If a hole is located at site 0 a virtual hop of the $f$ electron onto that lattice site creates a singly instead of a doubly 
occupied state. The kinetic energy of the $f$ electron is therefore increased. The last term shows that holes are weakly repelled from the neighboring sites $i$.

The term $H_{\mathrm{K}}$ is the analogue of the spin-spin interaction in the Kondo Hamiltonian

$$
H_{\mathrm{K}}=\frac{2 \gamma V^{2}}{U-\epsilon_{f}} \mathbf{S}_{f} \mathbf{S}_{0}-\frac{t V^{2}(2+r)}{U \epsilon_{f}(1+r)^{2}} \mathbf{S}_{f} \sum_{i(0)}\left(\hat{\mathbf{s}}_{i 0}+\hat{\mathbf{s}}_{0 i}\right),
$$

with $\hat{\mathbf{s}}_{0 i}=1 / 2 \sum_{\alpha \beta} \hat{c}_{i \alpha}^{\dagger} \boldsymbol{\sigma}_{\alpha \beta} \hat{c}_{0 \beta}$. Since site 0 is doubly occupied in the virtual state, the dominant prefactor of the first term is $2 V^{2} /\left(U-\epsilon_{f}\right)$ rather than $-2 V^{2} / \epsilon_{f}$ as in the case of uncorrelated electrons [cf. Eq. (3.1)]. The other terms arise from the application of $L_{t}$ and indicate a delocalization of the interaction.

Finally, there is a contribution $H^{\prime}$ to $\tilde{H}^{(\mathrm{h})}$, which describes an interaction specific to the strong correlation case

$$
H^{\prime}=\frac{2 V^{2} t^{2}}{U \epsilon_{f}^{2}(1+r)^{2}} \sum_{i(0)} \mathbf{S}_{f}\left[\mathbf{s}_{i}\left(1-n_{0}\right)-\mathbf{s}_{0}\left(1-n_{i}\right)\right]
$$

The first term implies an antiferromagnetic interaction between the impurity and the nearest neighbor sites of site 0 provided there is a hole at site 0 . Note that in the half-filled case the spins of the impurity and those of the nearest neighbor sites $i$ are ferromagnetically aligned. The second term can be considered as a correction to the Kondo-type interaction. When an electron at site 0 with spin $\mathbf{s}_{0}$ hops to an empty site $i$, the $f$ electron can hop virtually onto site 0 without creating a double occupancy. Hence, the antiferromagnetic spin exchange is decreased.

\section{B. Half-filled case}

In the case of half filling $n_{j}=1$ for all $j$, and the effective Hamiltonian (4.5) reduces to

$$
\begin{aligned}
H_{\mathrm{eff}}= & \Delta E+H_{\mathrm{H}}+H_{\mathrm{K}} \\
= & -\frac{Z t^{2} N_{\mathrm{S}}}{2 U}+\epsilon_{f}-\frac{\gamma V^{2}}{2\left(U-\epsilon_{f}\right)}-\frac{Z V^{2} t^{2}}{2 \epsilon_{f} U^{2}(1+r)} \\
& +\sum_{j x} J_{j} \mathbf{s}_{j} \mathbf{s}_{j+x}+\frac{2 \gamma V^{2}}{U-\epsilon_{f}} \mathbf{S}_{f} \mathbf{s}_{0},
\end{aligned}
$$


where $J_{j}$ is as in Eq. (4.8). $N_{\mathrm{S}}$ denotes the number of the lattice sites in the substrate. The conduction electrons are described by an Heisenberg antiferromagnet with a reduced Heisenberg exchange in the neighborhood of the impurity. The $f$ spin couples via an antiferromagnet spin exchange to the spin at the lattice site which is located nearest to the $f$ orbital. If the (weak) $j$ dependence of $J_{j}$ is neglected, the Hamiltonian (4.12) reduces to that of Ref. 15 .

\section{Electron doping}

Next, we treat the case of a system with more than half filling (electron doping). We proceed in close analogy to Sec. IVA, where the case of hole doping was discussed. In the limit of large $U$, empty sites are excluded here. Let $\tilde{P}_{c}$ denote the projector onto the configuration space without empty sites and $\tilde{Q}_{c}=1-\tilde{P}_{c}$. The effective Hamiltonian reduced to the space without empty sites reads [cf. Eq. (4.1)]

$$
\tilde{H}^{(\mathrm{e})}=\tilde{P}_{c} H_{\mathrm{eff}} \tilde{P}_{c}+\tilde{P}_{c} H_{\mathrm{eff}} \tilde{Q}_{c} \frac{1}{E^{(\mathrm{e})}-\tilde{Q}_{c} H_{\mathrm{eff}} \tilde{Q}_{c}} \tilde{Q}_{c} H_{\mathrm{eff}} \tilde{P}_{c}
$$

where $E^{(\mathrm{e})}$ is the ground-state energy. Only those parts of $H_{\text {eff }}$ contribute to the last term to order $U^{-1}$ which generate an empty site. Thus,

$$
\tilde{H}^{(\mathrm{e})}=\tilde{P}_{c} H_{\mathrm{eff}} \tilde{P}_{c}-\frac{1}{U} \tilde{P}_{c}\left(H_{t}+H^{(1)}\right) \tilde{Q}_{c}\left(H_{t}+H^{(1)}\right) \tilde{P}_{c}
$$

Again, we neglect terms of order $V^{4}$, as well as terms involving three lattice sites. Using the relation $\tilde{P}_{c} n_{j} \tilde{P}_{c}=1+n_{j \uparrow} n_{j \downarrow}$ and regrouping the different terms in $\tilde{H}^{(\mathrm{e})}$, we find

$$
\tilde{H}^{(\mathrm{e})}=\Delta E^{(\mathrm{e})}+H_{\mathrm{kin}}^{(\mathrm{e})}+H_{\mathrm{H}}^{(\mathrm{e})}+H_{\mathrm{p}}^{(\mathrm{e})}+H_{\mathrm{K}}^{(\mathrm{e})},
$$

where 


$$
\begin{aligned}
\Delta E^{(\mathrm{e})}= & \epsilon_{f}-\frac{\gamma V^{2}}{2\left(U-\epsilon_{f}\right)}+U\left(N_{\mathrm{c}}-N_{\mathrm{S}}\right) \\
H_{\mathrm{kin}}^{(\mathrm{e})}= & \sum_{j x, \sigma} T_{j}^{(\mathrm{e})}\left(\bar{c}_{j \sigma}^{\dagger} \bar{c}_{j+x \sigma}+\bar{c}_{j+x \sigma}^{\dagger} \bar{c}_{j \sigma}\right) \\
H_{\mathrm{H}}^{(\mathrm{e})}= & \sum_{j x} J_{j} \mathbf{s}_{j} \mathbf{s}_{j+x} \\
& -\sum_{j x} \frac{J_{j}}{4}\left(1-n_{j \uparrow} n_{j \downarrow}\right)\left(1-n_{j+x \uparrow} n_{j+x \downarrow}\right) \\
H_{\mathrm{p}}^{(\mathrm{e})}= & \frac{\gamma V^{2}}{2\left(U-\epsilon_{f}\right)} n_{0 \uparrow} n_{0 \downarrow} \\
H_{\mathrm{K}}^{(\mathrm{e})}= & \frac{2 \gamma V^{2}}{U-\epsilon_{f}} \mathbf{S}_{f} \mathbf{s}_{0}+\frac{t V^{2}}{\left(U-\epsilon_{f}\right)^{2}} \mathbf{S}_{f} \sum_{i(0)}\left(\overline{\mathbf{s}}_{0 i}+\overline{\mathbf{s}}_{i 0}\right) .
\end{aligned}
$$

$N_{\mathrm{S}}$ is the number of lattice sites in the substrate, $N_{\mathrm{c}}$ is the number of conduction electrons, so that there are $N_{\mathrm{c}}-N_{\mathrm{S}}$ doubly occupied sites, which contribute with an energy $U\left(N_{\mathrm{c}}-N_{\mathrm{S}}\right)$ to the energy shift $\Delta E^{(\mathrm{e})}$.

As in the case of hole doping, the second and third term in Eq. (4.15), $H_{\text {kin }}^{(e)}+H_{\mathrm{H}}^{(\mathrm{e})}$, define a $t-J$ model describing the conduction electrons with parameters, which are modified in the neighborhood of the impurity:

$$
\begin{aligned}
T_{j}^{(\mathrm{e})} & =-\frac{t}{2}\left(1-\frac{V^{2}}{2\left(U-\epsilon_{f}\right)^{2}} \delta_{j 0}\right) \\
J_{j} & =\frac{2 t^{2}}{U}\left(1-\frac{V^{2}}{\left|\epsilon_{f}\right|\left(U-\epsilon_{f}\right)} \delta_{j 0}\right) .
\end{aligned}
$$

In the case of electron doping, double occupancies hop rather than holes: $\bar{c}_{j \sigma}^{\dagger}=c_{j \sigma}^{\dagger} n_{j \bar{\sigma}}$. Since the $f$ electron can gain kinetic energy only if site 0 is singly occupied, double occupancies are repelled from site 0 . This is described by the "potential", $H_{\mathrm{p}}^{(\mathrm{e})}$. The Kondo-type interaction, $H_{\mathrm{K}}^{(\mathrm{e})}$, between the $f$ spin and the spin of the conduction electrons consists of two parts. The local part is the same as in the case of hole doping, cf. Eq. (4.10). The prefactor of the second term is modified and we introduced $\overline{\mathbf{s}}_{0 i}=1 / 2 \sum_{\alpha \beta} \bar{c}_{i \alpha}^{\dagger} \boldsymbol{\sigma}_{\alpha \beta} \bar{c}_{0 \beta}$. However, in the case of electron doping there are no interactions of the $f$ spin with the conduction electrons corresponding to $H^{\prime}$, cf. Eq. (4.11).

Finally, we mention that in the half-filled case $n_{j \uparrow} n_{j \downarrow}=0$, and the Hamiltonian (4.15) reduces to that of Eq. (4.12). 


\section{SUMMARY}

In this paper, we considered a magnetic impurity which interacts weakly by hybridization with a system of strongly correlated electrons. This contrasts the situation treated in the Anderson impurity model where the impurity interacts with free conduction electrons. We described the strong correlations among the conduction electrons by a Hubbard Hamiltonian and performed a canonical transformation to eliminate the charge degrees of freedom at the impurity site. For vanishing Hubbard repulsion $U$, this procedure was shown to be equivalent to the conventional Schrieffer-Wolff transformation and yields the Kondo Hamiltonian.

Of particular interest is the limit $U \gg t$. Here, we additionally reduced the charge degrees of freedom of the conduction electrons by a degenerate perturbation expansion which, in the absence of the magnetic impurity, leads to the $t-J$ model. The corrections we find are due to the impurity. In the case of half filling, the conduction electrons are described by a Heisenberg Hamiltonian with an exchange coupling constant, which is slightly modified in the neighborhood of the impurity. Furthermore, there is an exchange interaction between the impurity spin and the spin of the conduction electron at the lattice site closest to the impurity, i.e., site 0 . The coupling constant depends on $U$ and is, therefore, different than in the usual Kondo Hamiltonian.

When the system is doped new terms arise. Since we strictly forbid double occupancies

of the impurity site the electron-hole symmetry is broken and we have to treat the cases of hole and electron doping separately. If the system is doped with electrons, doubly occupied sites are repelled by the impurity since otherwise the exchange becomes impossible.

When we dope the system with holes, the conduction electrons move like in a $t-J$ model. The holes are attracted towards the impurity, which reflects the gain in kinetic energy if the $f$ electron hops without creating a doubly occupied site. Additionally, we find a new type of interaction: Provided a hole is located at the lattice site 0 there is an antiferromagnetic exchange of the impurity spin with the conduction electron spin at the nearest neighbor sites of 0 . In the undoped system these spins are ferromagnetically aligned. 


\section{APPENDIX A: EFFECTIVE HAMILTONIAN}

In the following, we derive the effective Hamiltonian (2.12) which is obtained from the starting Hamiltonian (2.4) after performing the canonical transformation (2.5) that eliminates the charge degrees of freedom of the $f$ orbital and projecting onto the space with

$n_{f}=1$. We begin by listing the terms to lowest order in the expansion (2.10) of $1 /\left(\epsilon_{f}-L_{c}\right)$. The zeroth-order term $(\nu=0)$ reads

$$
\frac{1}{\epsilon_{f}-L_{U}} c_{0 \sigma}^{\dagger}=\frac{1}{\epsilon_{f}}\left(1-\frac{1}{1+r} n_{0 \bar{\sigma}}\right) c_{0 \sigma}^{\dagger}
$$

For $\nu=1$ one obtains

$$
\begin{aligned}
\frac{1}{\epsilon_{f}-L_{U}} L_{t} \frac{1}{\epsilon_{f}-L_{U}} c_{0 \sigma}^{\dagger}= & \frac{-t}{\epsilon_{f}^{2}} \sum_{i(0)}\left[\left(1-\frac{1}{1+r} n_{i \bar{\sigma}}\right)\left(1-\frac{1}{1+r} n_{0 \bar{\sigma}}\right) c_{i \sigma}^{\dagger}\right. \\
& \left.-\frac{1}{1+r}\left(1-\frac{1}{1+r} n_{i \sigma}\right) c_{i \bar{\sigma}}^{\dagger} c_{0 \bar{\sigma}} c_{0 \sigma}^{\dagger}+\frac{1}{(1+r)^{2}}\left(r+n_{i \sigma}\right) c_{0 \bar{\sigma}}^{\dagger} c_{i \bar{\sigma}} c_{0 \sigma}^{\dagger}\right]
\end{aligned}
$$

and for $\nu=2$

$$
\frac{t^{2}}{\epsilon_{f}^{3}} \sum_{i(0)}\left[\left(1-\frac{(1+2 r)^{2}}{(1+r)^{3}} n_{0 \bar{\sigma}}+\frac{r}{(1+r)^{2}} n_{i \bar{\sigma}}\right) c_{0 \sigma}^{\dagger}-\frac{r}{(1+r)^{2}}\left(c_{i \bar{\sigma}}^{\dagger} c_{0 \bar{\sigma}} c_{i \sigma}^{\dagger}-c_{0 \bar{\sigma}}^{\dagger} c_{i \bar{\sigma}} c_{i \sigma}^{\dagger}\right)\right] .
$$

The summations $i(0)$ are restricted over nearest neighbor sites $i$ of site 0 and in Eq. (A3) we have neglected contributions from next-nearest neighboring sites. When the Eqs. (A1 A3) are set into Eq. (2.9) one obtains $H_{\text {eff }}$ in the form

$$
H_{\mathrm{eff}}=H_{c}+\epsilon_{f}+H^{(0)}+H^{(1)}+H^{(2)} .
$$

The different contributions are

$$
H^{(0)}=\frac{V^{2}}{\epsilon_{f}}-\frac{V^{2}(2+r)}{2 \epsilon_{f}(1+r)} n_{0}+\frac{V^{2}}{\epsilon_{f}(1+r)} n_{0 \uparrow} n_{0 \downarrow}+\frac{2 V^{2}}{U(1+r)} \mathbf{S}_{f} \mathbf{s}_{0},
$$




$$
\begin{aligned}
H^{(1)}= & \frac{t V^{2}}{4 \epsilon_{f}^{2}(1+r)^{2}} \sum_{i(0) \sigma}\left(c_{i \sigma}^{\dagger} c_{0 \sigma}+c_{0 \sigma}^{\dagger} c_{i \sigma}\right)\left(2+2 r+r^{2}-(2+r) n_{0 \bar{\sigma}}-(3+r) n_{i \bar{\sigma}}+3 n_{0 \bar{\sigma}} n_{i \bar{\sigma}}\right) \\
& +\frac{t V^{2}}{2 \epsilon_{f}^{2}(1+r)^{2}} S_{f}^{z} \sum_{i(0) \sigma}(-)^{\sigma}\left(c_{i \sigma}^{\dagger} c_{0 \sigma}+c_{0 \sigma}^{\dagger} c_{i \sigma}\right)\left(r(r+2)+(1-r) n_{i \bar{\sigma}}-r n_{0 \bar{\sigma}}-n_{0 \bar{\sigma}} n_{i \bar{\sigma}}\right) \\
& +\frac{t V^{2}}{2 \epsilon_{f}^{2}(1+r)^{2}} \sum_{i(0)}\left(S_{f}^{+} s_{i 0}^{-}+S_{f}^{-} s_{0 i}^{+}\right)\left(r(r+2)+(1-r) n_{i \uparrow}-r n_{0 \downarrow}-n_{0 \downarrow} n_{i \uparrow}\right) \\
& +\frac{t V^{2}}{2 \epsilon_{f}^{2}(1+r)^{2}} \sum_{i(0)}\left(S_{f}^{+} s_{0 i}^{-}+S_{f}^{-} s_{i 0}^{+}\right)\left(r(r+2)+(1-r) n_{i \downarrow}-r n_{0 \uparrow}-n_{0 \uparrow} n_{i \downarrow}\right),
\end{aligned}
$$

and

$$
\begin{aligned}
H^{(2)}= & \frac{Z V^{2} t^{2}}{\epsilon_{f}^{3}}-\frac{V^{2} t^{2}}{2 U \epsilon_{f}^{2}(1+r)^{2}} \sum_{i(0)}\left(c_{i \uparrow}^{\dagger} c_{i \downarrow}^{\dagger} c_{0 \downarrow} c_{0 \uparrow}+c_{0 \uparrow}^{\dagger} c_{0 \downarrow}^{\dagger} c_{i \downarrow} c_{i \uparrow}\right) \\
& -\frac{Z V^{2} t^{2}\left(2+7 r+7 r^{2}+r^{3}\right)}{2 \epsilon_{f}^{3}(1+r)^{3}} n_{0}-\frac{V^{2} t^{2}}{2 U \epsilon_{f}^{2}(1+r)^{2}} \sum_{i(0)} n_{i}+\frac{Z V^{2} t^{2}(1+2 r)^{2}}{\epsilon_{f}^{3}(1+r)^{3}} n_{0 \uparrow} n_{0 \downarrow} \\
& -\frac{V^{2} t^{2}}{U \epsilon_{f}^{2}(1+r)^{2}} \sum_{i(0)}\left(\mathbf{s}_{0} \mathbf{s}_{i}-\frac{n_{0} n_{i}}{4}\right) \\
& -\frac{V^{2} t^{2}}{U \epsilon_{f}^{2}(1+r)^{2}} \sum_{i(0)} \mathbf{S}_{f} \mathbf{s}_{0}\left(\frac{2\left(1+r-r^{2}\right)}{1+r}-n_{i}\right)+\frac{V^{2} t^{2}}{U \epsilon_{f}^{2}(1+r)^{2}} \sum_{i(0)} \mathbf{S}_{f} \mathbf{s}_{i}\left(2-n_{0}\right) .
\end{aligned}
$$

The Hamiltonian $H_{\text {eff }}$ is discussed in several limiting cases in Secs. III and IV. 


\section{REFERENCES}

${ }^{1}$ T. Brugger, T. Schreiner, G. Roth, P. Adelmann, and G. Czjzek, Phys. Rev. Lett. 71, $2481(1993)$.

${ }^{2}$ P. Fulde, J. Keller, and G. Zwicknagl, in Solid state physics, Vol. 41, p. 1 edited by H. Ehrenreich and D. Turnbell (Academic Press, San Diego, 1988).

${ }^{3}$ P. Fulde, V. Zevin, and G. Zwicknagl, Z. Phys. B 92, 133 (1993).

${ }^{4}$ S. Skanthakumar, H. Zhang, T. W. Clinton, W.-H. Li, J. W. Lynn, Z. Fisk, and S.-W. Cheong, Physica C $\underline{160}, 124$ (1989).

${ }^{5}$ S. B. Oseroff, D. Rao, F. Wright, D. C. Vier, S. Schultz, J. D. Thompson, Z. Fisk, S.-W. Cheong, M. F. Hundley, and M. Tovar, Phys. Rev. B. 41, 1934 (1990).

${ }^{6}$ A. T. Boothroyd, S. M. Doyle, D. McK. Paul, D. S. Misra, and R. Osborn, Physica C $\underline{165}$, 17 (1990).

${ }^{7}$ P. Adelmann, R. Ahrens, G. Czjzek, G. Roth, H. Schmidt, and C. Steinleiter, Phys. Rev. B $\underline{46}, 3619$ (1992).

${ }^{8}$ K. J. v. Szczepanski, T. M. Rice, and F. C. Zhang, Europhys. Lett. $\underline{8}, 797$ (1989).

${ }^{9}$ P. Rubin and A. Sherman, Phys. Stat. Sol. (b) 166, 161 (1991).

${ }^{10}$ K. M. Rabe and R. N. Bhatt, J. Appl. Phys. $\underline{69}, 4508$ (1991).

${ }^{11}$ N. Bulut, D. Hone, D. J. Scalapino, and E. Y. Loh, Phys. Rev. Lett. $\underline{62}, 2192$ (1989).

12 M. Acquarone and M. Paiusco, Physica C $\underline{210}, 373$ (1993).

${ }^{13}$ D. Poilblanc, W. Hanke, and D. J. Scalapino, Phys. Rev. Lett. $\underline{72}, 884$ (1994).

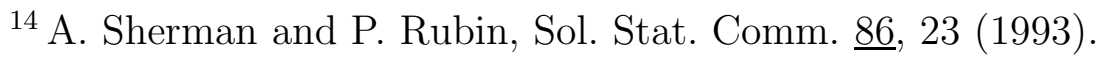

${ }^{15}$ N. Nagaosa, Y. Hatsugai, and M. Imada, J. Phys. Soc. Jpn. 토, 978 (1989). 


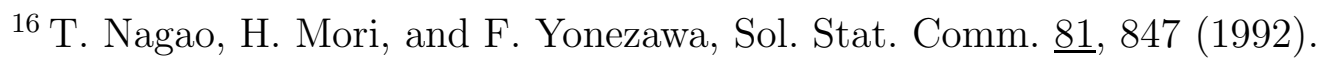

${ }^{17}$ P. W. Anderson, Phys. Rev. 124, 41 (1961).

18 J. R. Schrieffer and P. A. Wolff, Phys. Rev. 149, 491 (1966).

19 J. Kondo, Prog. Theo. Phys. $\underline{32}, 37$ (1964).

${ }^{20}$ T. Kato, Perturbation Theory for Linear Operators, (Springer Verlag, New York, 1965). 